\title{
Research on Simulation Programming of Image Transformation Based on Information
}

\author{
Binchao Xu \\ School of Physical Education, HeZHou University, Hezhou, China
}

\begin{abstract}
Keywords: information technology; physical education; information recognition; image transformation; simulation; change angle
\end{abstract}

\begin{abstract}
With the development of information network, information technology has been widely applied to many fields. Informatization promotes intelligence a lot of work and strengthens the working process of interaction, improving the work efficiency and work quality. In this context, this thesis carries on further research for the recognition of image and the transformation process of information technology, and establishes a mathematical model for action recognition and transformation process. The third part of the paper compiles the computer program of computer image motion recognition and transform, taking the basketball teaching as an example, and the design of the physical education multimedia information platform is discussed in the sports teaching process, the information platform is the use of the basketball movement design and development process. Finally, through the method of software window display, it obtains the change angle of basketball trunk body movements in 155s, to provide the theory reference for the informatization development of sports teaching process.
\end{abstract}

\section{Introduction}

Information technology is one of the most popular research techniques in twenty-first Century, which has been widely used in many engineering fields [1]. With the development of network information, more and more students use the network to learn, so the development and design of computer network learning network platform has become a hot research, this paper uses the computer application as background, the human body recognition and the design process of action are studied for the information technology, the first part of the thesis carries on the investigation for the popularization situation of the university computer network [2,3]; the second part of the thesis carries on the mathematical modeling for the recognition process of computer human body information, and then carries on algorithm program [4]. Finally, the basketball teaching practice as an example carries on the experimental research on the identification process of basketball movement information, and the use of VB numerical value simulation software obtains the basketball action's design chart and parametric curve, to provide the computer data support for the study of basketball teaching.

\section{The research status of university computer information technology}

Before the research of university computer information technology, the popularization of computer network information is studied. This paper selects the south of a university as the research object, the use situation of computer network information is carried on research, and finally the results are shown in Figure 1.

It can be seen from Figure 1 that about $61.1 \%$ of the students use the Internet number is greater than $8 \mathrm{~h}$, the Internet number is less than $5 \mathrm{~h}$ accounted for only $11.2 \%$, and online number is in the $5 \mathrm{~h}$ to $8 \mathrm{~h}$ accounted for about $27.7 \%$. In Figure 1, we can be seen that universities are higher for the rate of using network, and the network utilization rate is not high [5]. Many students are addicted to chat and games for the use of network information, however the use of learning network is not much. Especially the weekend time is lower for network utilization rate.

In view of this situation, this paper takes the networked teaching idea as the instruction, to carry on the design of sports teaching and practice network teaching platform system [6,7]. Firstly, the 
human motion information recognition process is established in the process of physical education teaching, and then we carry out programmed according to the mathematical model.

\section{Computer human motion information recognition process}

As shown in Figure 2, the ground or space point $P$ is along the ellipsoidal normal direction that is projected elliptical surface point for the $P_{0}$, the position vectors are respectively said by $\vec{R}$ and $\vec{R}_{0}$, the height of $P$ point is $H$. A unit vector of normal is set to $\vec{n}$, and then the position of $P$ point is the sum of two vectors, i.e.

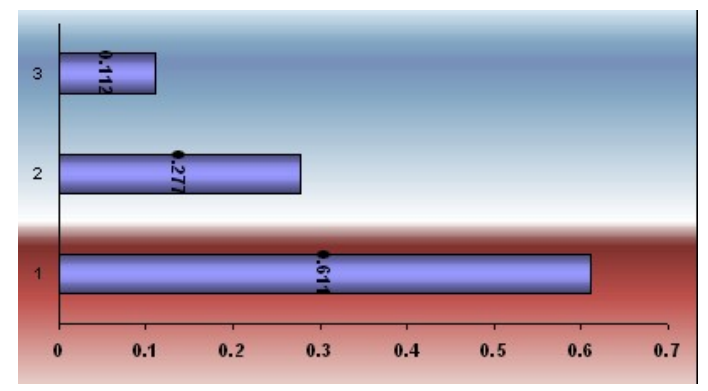

Figure 1. The investigation of network information penetration

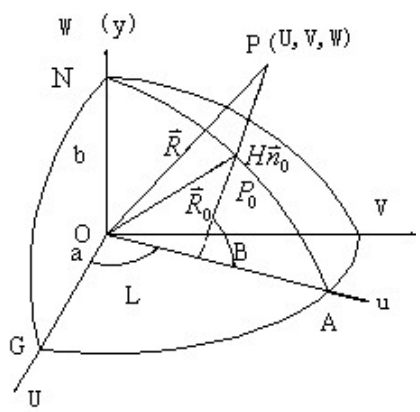

Figure 2. Computer body identify coordinate system

Then we can get the formula (1).

$$
\vec{R}=\vec{R}_{0}+H \vec{n}
$$

Through the meridian plane NOA Cartesian coordinates $\mathrm{O}-\mathrm{xy}$, the expression of position vector $\vec{R}_{0}$ can get the formula (2).

In which,

$$
\left.\begin{array}{l}
u=\frac{a}{m} \cos B \\
v=\frac{a}{m}\left(1-e^{2}\right) \sin B
\end{array}\right\}
$$

$$
m=\sqrt{1-e^{2} \sin ^{2} B}
$$

$\vec{R}_{0}$ can be written as:

$$
\vec{R}_{0}=\left|\begin{array}{c}
u \cos L \\
u \sin L \\
v
\end{array}\right|=\frac{a}{m}\left|\begin{array}{c}
\cos B \cos L \\
\cos B \sin L \\
\sin B\left(1-e^{2}\right)
\end{array}\right|=N\left|\begin{array}{c}
\cos B \cos L \\
\cos B \sin L \\
\sin B\left(1-e^{2}\right)
\end{array}\right|
$$

In formula (4), $\frac{a}{m}=N$. From Figure 2, we can know that unit normal vector can be obtained

$$
\vec{n}=\left|\begin{array}{c}
\cos B \cos L \\
\cos B \sin L \\
\sin B
\end{array}\right|
$$

The two type of above formula (4) and formula (5) is substituted into the formula (6), we can get

$$
\vec{R}=\left|\begin{array}{l}
X \\
Y \\
Z
\end{array}\right|=\left|\begin{array}{c}
(N+H) \cos B \cos L \\
(N+H) \cos B \sin L \\
\left(N+H-N e^{2}\right) \sin B
\end{array}\right|
$$

In formula (6), its expansion can obtain $B, L$ and $H$ that can compute $U, V$ and $W$ formula. Inverse formula can be obtained by formula (7), the results are as follows: 


$$
\left.\begin{array}{l}
\operatorname{tg} L=\frac{V}{U} \\
\operatorname{tg} B=\frac{W+N e^{2} \sin B}{\sqrt{U^{2}+V^{2}}} \\
H=\sqrt{U^{2}+V^{2}} \sec B-N
\end{array}\right\}
$$

Because the source coordinate net shape remains unchanged, this method is also called the similarity transformation method. The transformation equations is

$$
\left.\begin{array}{l}
u=a+k\left(u^{\prime} \cos \alpha+v^{\prime} \sin \alpha\right) \\
v=b+k\left(-u^{\prime} \sin \alpha+v^{\prime} \cos \alpha\right)
\end{array}\right\}
$$

In formula (8), vector $a$ and $b$ show translation, $\alpha$ the source coordinate net $x$ axis reversal to the angle of target coordinate net $x$, and $k$ is the scale factor.

According to the least square method, the formula (8) must be linearization, to present a new parameter is $c$ and $d$

Formula (9) can be converted to

$$
c=k \cos \alpha, d=k \sin \alpha
$$

$$
\left.\begin{array}{l}
x=a+x^{\prime} c+y^{\prime} d \\
y=b+y^{\prime} c-x^{\prime} d
\end{array}\right\}
$$

\section{The application of computer information technology in the sports teaching process}

Coordinate class inherits the CObject class, it has four parameters' constructed function and a default constructor function for the easy storage, to realize the initialization of internal data. A structure stores the coordinates of the data, three pointer variables store the relevant information of coordinate data [8,9]. In this paper, through the VB numerical simulation software, we can analyze research on sports teaching practice, and the design and development of the main program process of information physical education teaching platform is as follows:

\#include "math.h"

\#if !defined(AFX_COORDINATE_H_92D7D474_43B6_40EB_9392_428F6F8EBA6E_INCLUDED_)

\#define AFX_COORDINATE_H_95D7D474_43B6_40EB_9391_428F6F8EBA6E_INCLUDED_

\#define A_B_C 0

\#define U_V_W 1

\#define u_v_w 2

typedef struct

\{

double u,v,w;

CString name, remark

\}COORDINATE;

typedef struct

\{

double du,dv,ratio,revolve;

double east,north,center;

CString name;

class CCoordinate: public CObject

\{

Public:

int $*$ x_format;

EParameter *x_parameter;

PROJECTION *x_projection;

Public:

COORDINATE x_coordinate;

CCoordinate();

CCoordinate(EParameter *parameter,int *format);

CCoordinate(EParameter * parameter,PROJECTION * projection,int *format);

CCoordinate(EParameter * parameter,int *format, COORDINATE * coordinate);

CCoordinate(EParameter*parameter,PROJECTION*projection,int*format,COORDINATE

public:

virtual $\sim$ CCoordinate()

\{\} ;

void UVW_ABC(COORDINATE e,COORDINATE *b);

void ABC _ UVW (COORDINATE e,COORDINATE *b);

void uvc_A ABC (COORDINATE e,COORDINATE *b);

\}

void ABC _ uvc (COORDINATE e,COORDINATE *b);

\#endif // !defined(AFX_COORDINATE_H_92D7D474_43B6_40EB_9392_428F6F8EBA6E_INCLUDED_) 
In this paper, human action recognition process of physical education practice is established by the program, and then the numerical simulation programming is established for by VB numerical simulation program, the sports teaching video transmission mainly uses for the following network transmission.

It can be seen from Figure 3, the information process of PE teaching mainly depends on the network transmission and computer image processing [10]. The computer carries on transmission image information through the image processing, and then it is transferred to the student client by the line teaching experiment room.
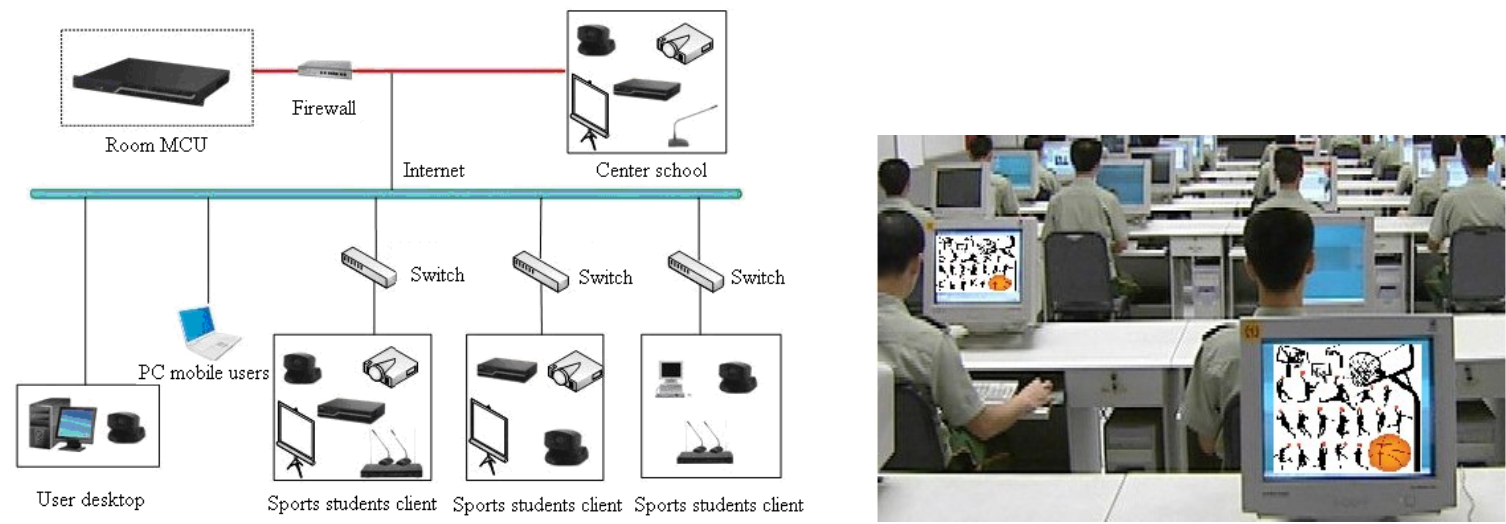

Figure 3. Schematic diagram of informatization process Figure 4. School client information system

Figure 4 shows the client information system, we can be seen from the chart that it is the sports teaching pattern [11]. In the students' client, teaching is transferred to student client computer by the design of basketball movement, to carry on the exchange of learning that has reached the principles of interactive teaching demonstration.

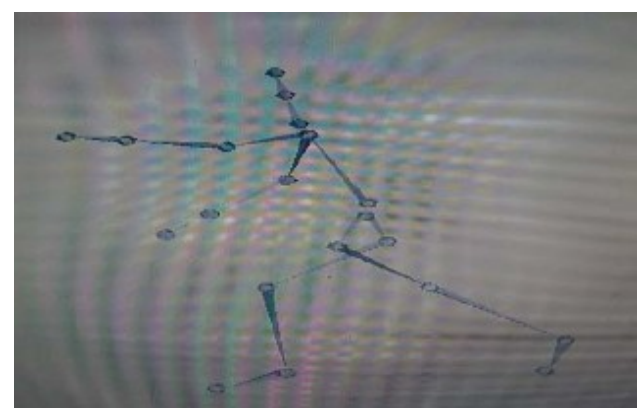

Figure 5. Basketball movement design

Figure 5 shows the action design of basketball teaching process, it can be seen from the chart that we can obtain the schematic diagram of a series of body movements for the basketball sports teaching action segmentation. Through the human body segmentation image normalization, we can obtain human basketball action demonstration graph as shown in Figure 6.

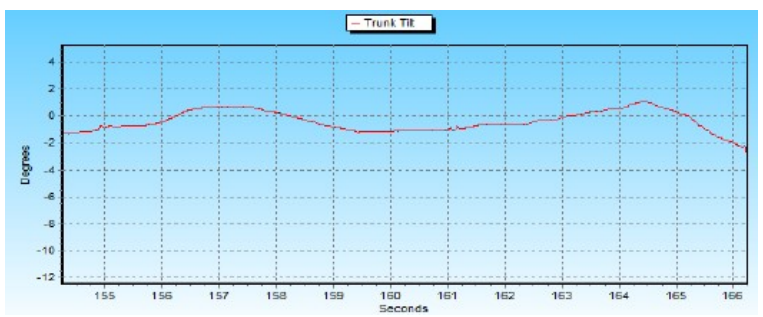

Figure 6. Basketball human action of computer design curve

Figure 6 shows the basketball human action computer design of VB curve simulation results, and it can be seen from the chart that the guidance of basketball movement provides the movement of the body different aspects at different time, and the range of change is fluctuation between 2 degrees and negative 4 degrees, they is very image of depicting the basketball shooting process.

\section{Conclusion}

Based on the information network technology, this paper carries on the in-depth analysis of the process of information human motion recognition, and taking the process of basketball teaching 
practice as an example, the application of sports teaching information technology is analyzed, finally combined with VB general numerical simulation software, basketball shooting is studied by numerical simulation. The first part of the thesis carries out research on the level of information network, and we found that universities are higher for the network popularization rate through research, and however the network utilization rate is not high. The second part of the thesis establishes the human motion information identification model, and according to the mathematical model, we has compiled the VB debugger, finally taking the basketball movement design as an example, the trunk change angle of basketball shooting process carries on simulation. Through the simulation, we can be seen that the guidance of basketball movement's body movement angle is different at different time, and the range of its variation is the fluctuation between 2 degree and negative 4 degrees, which is very image of depicting the basketball shooting process.

\section{References}

[1] He Kekang. The theory of information technology and curriculum deep integration [M]. Beijing: Beijing Normal University press, 2010: 112-132.

[2] Liu Chengxin. Integration and reconstruction -- interactive analysis of technology and classroom [M]. Beijing: Publishing House of electronics industry, 2011: 156-172.

[3] Zhang Lirong. The reflection and reconstruction of the relationship between information technology and education [M]. Beijing: Education Science Press, 2012: 89-95.

[4] Zhong Zhixian. The information teaching mode - theory construction and practice [M]. Beijing: Educational Science Publishing House, 2010: 312-320.

[5] Zhang Xuelin. Research on the innovation of university sports teaching method [J]. Journal of Liaoning public security and judicial administrative college, 2011 (2): 78-79.

[6] Hu Min. The status and optimization of physical education teaching methods classification system [J]. Hubei sports science and technology, 2010,29 (2): 176-177.

[7] Su Jian. Design of modern sports teaching methods principles and requirements [J]. Journal of forest resources education college, 2011,25 (4): 40-42.

[8] Jiang Hongxia. Chemistry concept map teaching research based on the information technology [D]. Chengdu: Sichuan Normal University, 2010: 3-10.

[9] Ren Jianhua, Ran Ming. Application of full interactive intelligent chemistry courseware and implementation method [J]. Chemistry teaching, 2006 (9): 47-49.

[10] Huang Hongmei. Application of interactive intelligent simulation courseware in the exploration of chemistry Informatization Instruction design [M]. Chengdu: Sichuan Normal University, 2011: 103-110.

[11] He Jialian. The study of chemical experiment research activities research based on information technology [D]. Sichuan Normal University, 2011: 2-15. 